New Cosmological Data and the Values of the Fundamental Parameters

ASP Conference Series, Vol. 666, 2001

A. Lasenby and A. Wilkinson, eds.

\title{
Type Ia Supernovae and the Value of the Hubble Constant
}

\author{
Brad K. Gibson, Chris B. Brook \\ Centre for Astrophysics \& Supercomputing, Swinburne University, Mail \\ \#31, P.O. Box 218, Hawthorn, Victoria, 3122 Australia
}

\begin{abstract}
The methodology involved in deriving the Hubble Constant via the calibration of the corrected peak luminosities of Type Ia supernovae $(\mathrm{SNe})$ is reviewed. We first present a re-analysis of the CalánTololo (C-T) and Center for Astrophysics (CfA) Type Ia SN surveys. Bivariate linear least squares and quadratic boot-strapped fits in peak apparent magnitude and light curve shape are employed to correct this heterogeneous sample of peak apparent magnitudes, resulting in an homogeneous (and excellent) secondary distance indicator: the so-called corrected peak luminosity. We next provide an empirical calibration for this corrected luminosity, using Cepheid-based distances for seven nearby spiral galaxies host to Type Ia SNe. Included in this sample is the spectroscopically peculiar SN 1991T (in NGC 4527), whose corrected peak luminosity is shown to be indistinguishable from that of so-called "normal" SNe. A robust value of the Hubble Constant is derived and shown to be $\mathrm{H}_{0}=73 \pm 2(\mathrm{r}) \pm 7(\mathrm{~s}) \mathrm{km} \mathrm{s}^{-1} \mathrm{Mpc}^{-1}$.
\end{abstract}

\section{Introduction}

The maximum brightness of a Type Ia supernova (SN) is not a standard candle. As illustrated graphically by Hamuy et al. (1996) and Riess et al. (1996), there is an order of magnitude span seen in the luminosities at the peak of the $\mathrm{SNe}$ light curves in their respective samples (depending upon the bandpass in question). On the other hand, an outstanding standard candle can be constructed by applying a light curve shape-dependent correction factor to the observed peak magnitude (e.g., Phillips et al. 1999; Jha et al. 1999; Suntzeff et al. 1999; Gibson et al. 2000; Freedman et al. 2001; Gibson \& Stetson 2001). This corrected peak luminosity succeeds as a standard candle where others fail because (i) the extreme luminosity of Type Ia SNe allows one to probe substantially further into the unperturbed Hubble flow (in comparison with competing secondary indicators such as the Tully-Fisher relation or surface brightness fluctuations), and (ii) the intrinsic scatter is smaller than that of other indicators.

Calibrating this secondary distance indicator can be approached from a purely theoretical tack (e.g., Höflich \& Khokhlov 1996), but the numerous model uncertainties still lead the conservative to favour the empirical calibration. The Sandage/Tammann/Saha Type Ia SN HST Calibration Project (Saha et al. 1999) was designed to provide just such a calibration, by determining the Cepheid distances to eight nearby galaxies (IC 4182, NGC 5253, 4536, 4496A, 
4639, 3627, 3982, 4527) host to Type Ia SNe. This original program has been supplemented with two additional calibrators situated within NGC 3368 (Tanvir et al. 1999) and NGC 4414 (Turner et al. 1998). Table 1 of Gibson et al. (2000) provides a complete list of the galaxies and SNe covered by all these calibration programs, including a subjective quality ranking.

In what follows, we review the steps involved in calibrating the Type Ia SNe extragalactic distance scale. We first provide a consistent set of linear and quadratic fits to the peak magnitude-light curve shape data from the CalánTololo (Hamuy et al. 1996) and Centre for Astrophysics (Riess et al. 1998,1999) SNe samples. These fits are then shown to provide a robust measure of the Hubble Constant when the relations are calibrated using the seven best (currently available) nearby Type Ia SNe with Cepheid-based host galaxy distance determinations.

\section{Constructing a Standard Candle}

If the peak luminosity of Type Ia SNe were a standard candle, one would expect the dispersion of the linear Hubble Diagram (magnitude versus redshift) to be limited only by photometric precision, providing both a precise and accurate value for the Hubble Constant $\mathrm{H}_{0}$ (once the zero point was set by calibrating the peak luminosity via nearby SNe of known distance). Combining the 50 Type Ia SNe円 in the Calán-Tololo (C-T: Hamuy et al. 1996) and Center for Astrophysics (CfA: Riess et al. 1998,1999) samples, Freedman et al. (2001; Fig 4) show that the raw B-, V-, and I-band Hubble Diagrams are anything but indicative of a standard candle (e.g., the dispersion in the B-band corresponds to a dispersion in distance (and $\mathrm{H}_{0}$ ) in excess of $40 \%$ ). Even upon applying corrections for foreground and host galaxy reddening, and culling the C-T+CfA samples for lowredshift interlopers (which are more susceptible to peculiar velocity corrections) and highly-reddened $\mathrm{SNe}$, the resulting dispersion and structure seen in the Hubble Diagrams (middle column of Fig 4 of Freedman et al. 2001) still indicate that an adequate standard candle is not at hand. To provide a useful constraint on $\mathrm{H}_{0}$, it is clear that these raw Hubble Diagrams need modification.

As has been well-documented over the past five years (e.g., Hamuy et al. 1996; Riess et al. 1996), the necessary modification is provided by applying a mild correction to the observed peak magnitudes of the Type Ia SNe, the size of the correction being a function of the shape of the light curve. In its simplest form (Hamuy et al. 1996), the observed peak magnitude $\mathrm{m}_{\max }$ (after correction for line-of-sight reddening) is modified by a linear function of the SN light curve decline rate $\Delta m_{15}$, where the latter is a measure of the B-band decline (in magnitudes) from the peak of the light curve to 15 days after the peak. Higherorder quadratic fits have also been employed on occasion (e.g., Suntzeff et al. 1999; Phillips et al. 1999; Gibson et al. 2000), as have more sophisticated multicolour light curve shape techniques (Riess et al. 1996,1998,1999). All such modifications result in Hubble Diagram dispersions which correspond to $\sim 7 \%$

\footnotetext{
${ }^{1} \mathrm{SN} 1996 \mathrm{ab}$ is not considered in what follows as its redshift is in excess of the regime over which
} the $k$-corrections employed by Hamuy et al. (1996) are applicable. 
in distance, and are consistent with that expected from the photometric errors alone.

Starting with Mark Phillips' (2000) homogeneous re-analysis of the photometry and light curves for both the C-T and CfA SNe samples, we construct our desired B-, V-, and I-band Hubble Diagrams by enforcing two strict criteria: the $\mathrm{SNe}$ must be consistent with (i) $3.5<\log (c z)_{\mathrm{CMB}}<4.5$, and (ii) $\left|B_{\max }-V_{\max }\right| \leq 0.20$. This yields a preferred subset of $36 \mathrm{SNe}$ for the B- and V-band Hubble Diagrams, and $32 \mathrm{SNe}$ for the I-band. Criterion (i) is (essentially) universally adopted (for reasons already noted previously), and (ii) was chosen to mimic that employed by Hamuy et al. (1996); stricter colour cuts have also been used (e.g., Phillips et al. 1999), but they have little impact on the science which follows.

Fits in decline rate $\Delta m_{15}$ and peak magnitude $m_{\max }$ are then constructed, the functional forms for which are

$$
\mathrm{m}_{\max }-\mathrm{R}_{\mathrm{m}} \mathrm{E}(\mathrm{B}-\mathrm{V})-5 \log (c z)=c\left(\Delta m_{15}-1.1\right)^{2}+b\left(\Delta m_{15}-1.1\right)+a
$$

where $R_{m}$ is the assumed colour-dependent ratio of total-to-selective absorption, $\mathrm{E}(\mathrm{B}-\mathrm{V})$ is the total line-of-sight reddening to a $\mathrm{SN}$, and $c z$ is its recessional velocity in the cosmic microwave background $(\mathrm{CMB})$ reference frame. The total line-of-sight reddening is decomposed into a Galactic foreground $\mathrm{E}(\mathrm{B}-\mathrm{V})_{\mathrm{G}}$ and host galaxy $\mathrm{E}(\mathrm{B}-\mathrm{V})_{\mathrm{H}}$ term:

$$
\mathrm{E}(\mathrm{B}-\mathrm{V})=\mathrm{E}(\mathrm{B}-\mathrm{V})_{\mathrm{G}}+\mathrm{E}(\mathrm{B}-\mathrm{V})_{\mathrm{H}}
$$

where $\mathrm{E}(\mathrm{B}-\mathrm{V})_{\mathrm{G}}$ is provided by the COBE/DIRBE dust maps of Schlegel et al. (1998) and $\mathrm{E}(\mathrm{B}-\mathrm{V})_{\mathrm{H}}$ is taken from Table 2 of Phillips et al. (1999). Prior to deriving the fit coefficients $a, b$, and $c$ for equation (1), extinction-dependent corrections to the decline rate (after Phillips et al. 1999) are applied, the form for which are

$$
\Delta m_{15}=\Delta m_{15}(\mathrm{raw})+0.1\left[\mathrm{E}(\mathrm{B}-\mathrm{V})_{\mathrm{G}}+\mathrm{E}(\mathrm{B}-\mathrm{V})_{\mathrm{H}}\right]
$$

Figure 1 shows our culled sample of $36 \mathrm{SNe}$ (32 in the I-band, recall) in the peak magnitude-decline rate plane, after the extinction corrections of equations (2) and (3) have been applied. A full accounting of the errors in photometry, extinction, and CMB velocity - assumed to be $\pm 600 \mathrm{~km} \mathrm{~s}^{-1}$ - (after Hamuy et al. 1996, Phillips et al. 1999, and Gibson et al. 2000) was made.

A bootstrapped, quadratic, outlier-resistant, fit to the data in each panel (B-, V-, and I-band, from top to bottom) was then undertaken, employing the functional form described by equation (1). The resultant colour-dependent coefficients $(a, b$, and $c)$, their associated uncertainties, and overall dispersion $\sigma$ are shown under the heading "Quadratic" in Figure 1. A bivariate linear least squares fit was also employed (effectively forcing coefficient $c$ of equation (1) to be zero), the results for which are also shown in Figure 1 adjacent to the heading "Linear". While $\sigma_{\text {quad }}<\sigma_{\text {lin }}$, the statistical significance of the improved fit is marginal (as measured by the Fisher F-test), and is the primary reason why, for example, Freedman et al. (2001) and Gibson \& Stetson (2001) reverted to linear fits subsequent to our original quadratic analysis (Gibson et al. 2000). 


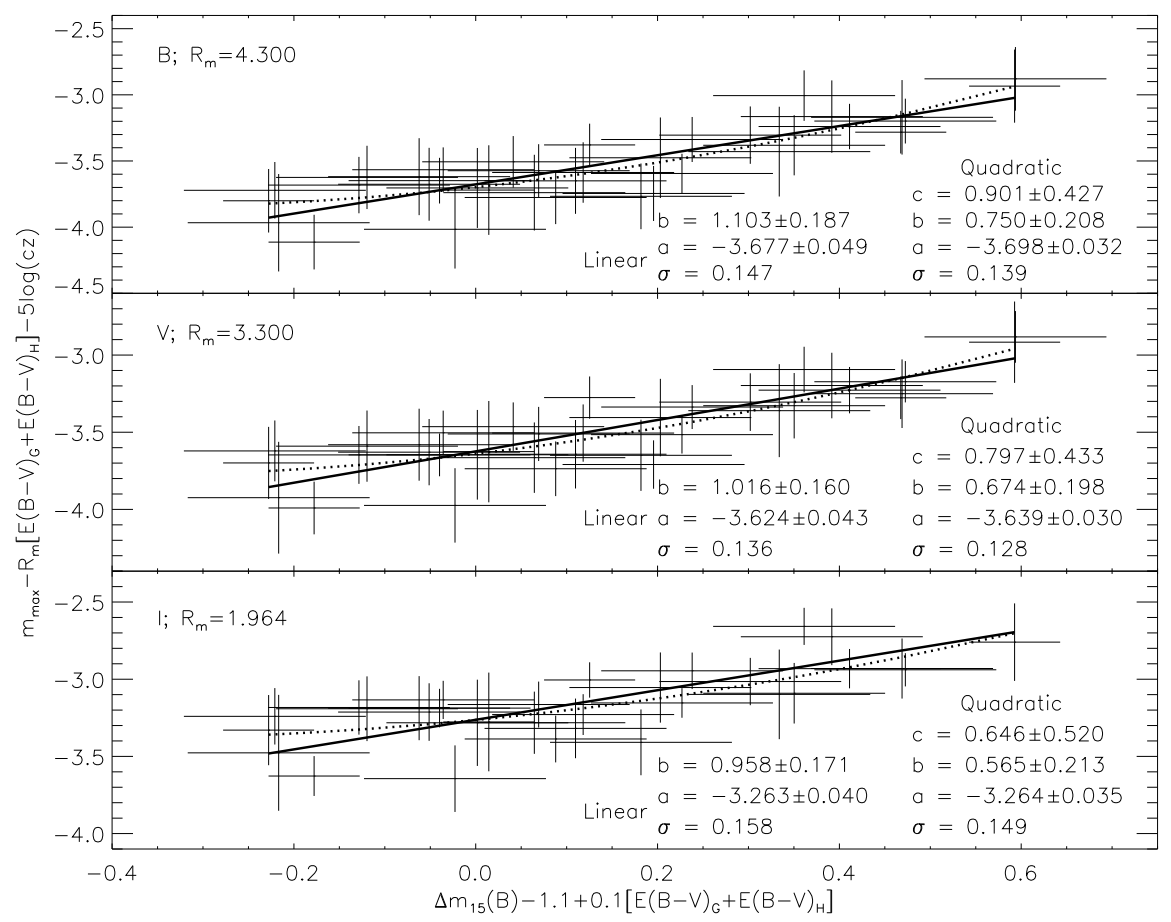

Figure 1. Peak magnitude $m_{\max }$ versus decline rate $\Delta m_{15}$ relationship for the subsample of C-T and CfA SNe with $3.5<\log (c z)_{\mathrm{CMB}}<4.5$ and $\left|\mathrm{B}_{\max }-\mathrm{V}_{\max }\right| \leq 0.20$. Foreground $\mathrm{E}(\mathrm{B}-\mathrm{V})_{\mathrm{G}}$ and host galaxy reddening corrections have been applied, after Schlegel et al. (1998) and Phillips et al. (1999), respectively, assuming ratios of total-to-selective absorption of $\mathrm{R}_{\mathrm{B}}: \mathrm{R}_{\mathrm{V}}: \mathrm{R}_{\mathrm{I}}=4.300: 3.300: 1.964$. The velocities $c z$ are in the $\mathrm{CMB}$ reference frame. Overlaid in each panel are the best quadratic (dotted) and linear (solid) fits to the data; coefficients, their uncertainties, and the resulting dispersion about the fit are likewise noted.

Regardless, adopting the quadratic fits in lieu of the linear ones has no impact upon the ultimately derived value of the Hubble Constant.

A wide variety of fits were undertaken, above and beyond the canonical quadratic and linear fits described above, including SNe subsets comprised of C-T SNe alone, replacing the Schlegel et al. (1998) foreground extinction maps with the classical Burstein \& Heiles (1982) values, reducing the recessional velocity uncertainty from $\pm 600 \mathrm{~km} \mathrm{~s}^{-1}$ to (somewhat arbitrarily) $\pm 300 \mathrm{~km} \mathrm{~s}^{-1}$, and modifying the ratios of total-to-selective absorption from

$\mathrm{R}_{\mathrm{B}}: \mathrm{R}_{\mathrm{V}}: \mathrm{R}_{\mathrm{I}}=4.300: 3.300: 1.964$ to $\mathrm{R}_{\mathrm{B}}: \mathrm{R}_{\mathrm{V}}: \mathrm{R}_{\mathrm{I}}=4.100: 3.100: 1.845$ (the latter being the ratios employed by Hamuy et al. 1996, Suntzeff et al. 1999, and Phillips et al. 1999). All permutations led to (essentially) identical values for the Hubble Constant, and so the reader will be spared the painful details. 


\section{Deriving the Hubble Constant}

Having generated what appears to be an excellent standard candle (the corrected peak luminosity of a Type Ia SNe), limited now only by photometric precision, we are left with the set of coefficients $a, b$, and (in the case of quadratic fits) $c$ for equation (1), but no immediate value for the Hubble Constant. The problem lies in the fact that the corrected peak luminosity is indeed an excellent secondary distance indicator (i.e., the corrected Hubble Diagrams provide accurate relative distances), but it still needs to be calibrated with nearby SNe of known distance $d$ (and therefore known luminosity $\mathrm{M}_{\max }$ ). Specifically, with equation (1) and some basic ASTRO 101:

$$
\begin{aligned}
\mathrm{M}_{\max } & =\mathrm{m}_{\max }-5 \log d-25 \\
c z_{\mathrm{CMB}} & =\mathrm{H}_{0} d
\end{aligned}
$$

one is left with a set of colour-dependent quadratic (or linear) equations relating the Hubble Constant $\mathrm{H}_{0}(\mathrm{~B}, \mathrm{~V}, \mathrm{I})$ to the peak absolute magnitude $\mathrm{M}_{\mathrm{B}, \mathrm{V}, \mathrm{I}}^{\max }$ :

$$
\mathrm{H}_{0}(\mathrm{~B}, \mathrm{~V}, \mathrm{I})=f\left(\mathrm{M}_{\mathrm{B}, \mathrm{V}, \mathrm{I}}^{\max }\right) .
$$

Thanks primarily to the efforts of the Sandage/Saha Type Ia SN HST Calibration Project (Saha et al. 1999), accurate Cepheid-based distances can now be derived for ten nearby galaxies which play host to well-observed Type Ia SNe. The Sandage/Saha program includes IC 4182 and NGCs 5253, 4536, 4496A, $4639,3627,3982$, 2 and 4527 , and has been supplemented with two additional calibrators situated within NGC 3368 (Tanvir et al. 1999) and NGC 4414 (Turner et al. 1998). Table 1 below lists the galaxy and SN name for the seven highestquality peak luminosity calibrators; it is these seven which provide the $M_{B, V, I}^{\max }$ calibration employed in determining $\mathrm{H}_{0}$ via equation (4).

The data reduction, Cepheid identification, and PL fitting for all but one of the above galaxies (NGC 4527) has already been presented in Gibson et al. (2000), and so will not be replicated here. Suffice it to say that the data were processed with ALLFRAME (instrumental photometry - Stetson 1994) and TRIAL (calibration and variable finding - Stetson 1996), following the precepts laid out earlier by the HST Key Project on the Extragalactic Distance Scale. The WFPC2 photometric zero point and charge-transfer corrections used here are based on Stetson (1998), "3 which differs from the Gibson et al. (2000) analysis where an a posteriori systematic transformation to the Hill et al. (1998) zero point was applied. The justification for adopting the Stetson (1998) calibration has been presented previously (Freedman et al. 2001; Gibson \& Stetson 2001).

Two further modifications to the Gibson et al. (2000) analysis are (i) the adoption of an LMC true modulus of $18.45 \pm 0.10$, and (ii) the LMC apparent PL relations of Udalski et al. (1999). The justification for both are provided by Freedman et al. (2001); both (i) and (ii) result in systematic downward shifts in derived galaxy distance, of $0.05 \mathrm{mag}$ and (of order) $0.16 \mathrm{mag}$, respectively. The

\footnotetext{
${ }^{2}$ Data proprietary at the time of writing.

${ }^{3}$ Which are nearly identical to those of Whitmore et al. (1999) and Dolphin (2000).
} 
Udalski et al. (1999) I-band PL relation is $\sim 0.10 \mathrm{mag} \mathrm{dex}^{-1}$ flatter in slope than that claimed by Madore \& Freedman (1991), and is to be preferred over the latter (Freedman et al. 2001).

Since the Gibson et al. (2000) analysis, Gibson \& Stetson (2001) have analysed NGC 4527, host galaxy to the spectroscopically peculiar SN 1991T. Sixteen high-quality Cepheids were identified in the dataset, PL fitting undertaken, and a true distance modulus of $30.482 \pm 0.085$ derived (neglecting any putative metallicity-dependent modifications to the Cepheid PL relations). Figure 2 shows the V- and I-band PL relations for the NGC 4527 Cepheids (upper two panels), and the distribution of individually de-reddened distance moduli (lower panel).

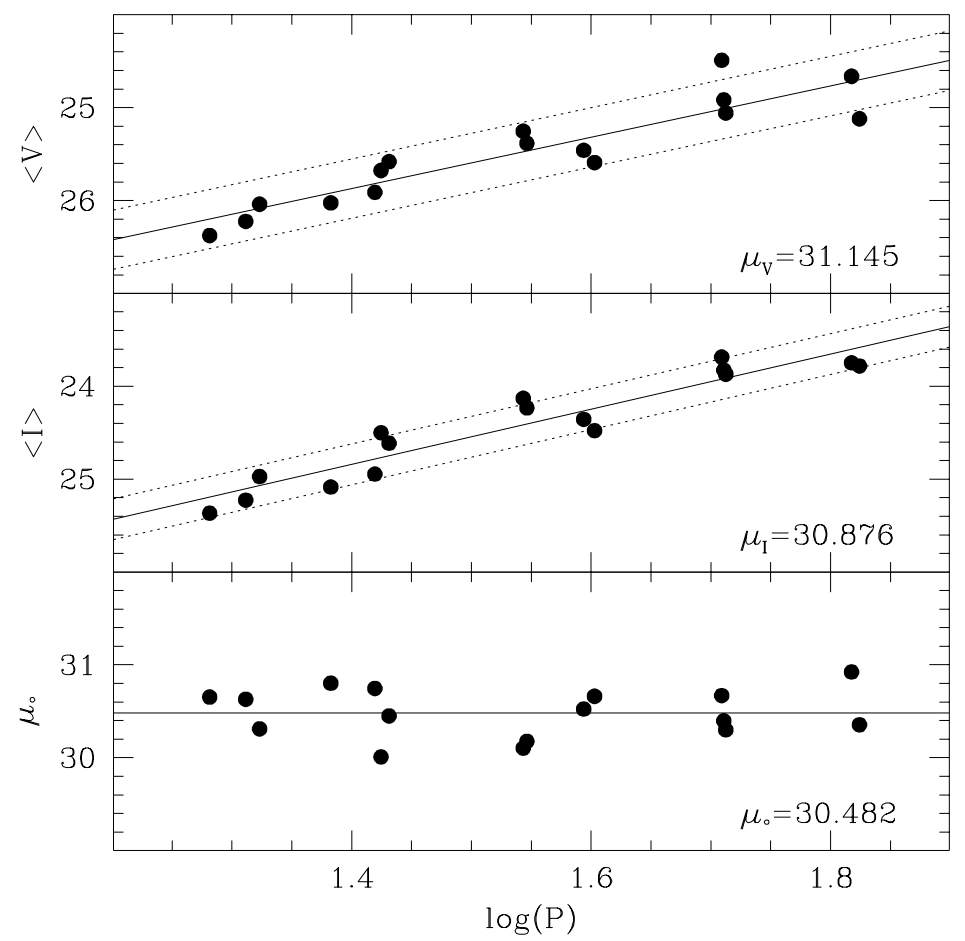

Figure 2. Apparent period-luminosity relations in the $V$ - (upper panel) and I-bands (middle panel) based upon the 16 high-quality Cepheid candidates discovered in NGC 4527 (Gibson \& Stetson 2001). The solid lines are least-squares fits to this entire sample, with the slope fixed to be that of the Udalski et al. (1999) LMC PL relations, while the dotted lines represent their corresponding $2 \sigma$ dispersion. The inferred apparent distance moduli, ignoring metallicity effects, are then $\mu_{\mathrm{V}}=31.145 \pm 0.056$ (internal) and $\mu_{\mathrm{I}}=30.876 \pm 0.046$ (internal). Lower Panel: Distribution of individually de-reddened Cepheid true moduli, as a function of period. The mean corresponds to $\left\langle\mu_{\circ}\right\rangle=30.482 \pm 0.066$ (internal: $\pm 0.085 \mathrm{mag}$, incorporating all random uncertainties - after entry $R_{P L}$ in Table 7 of Gibson et al. 2000).

Table 1 summarises the results of our (Gibson \& Stetson 2001) recent reanalysis of the extant HST WFPC2 data for the host galaxies of the seven high- 
quality nearby Type Ia SNe calibrators. Re-fitting of Cepheid PL relations with $\left(\mu_{\mathrm{Z}}\right)$ and without $\left(\mu_{\circ}\right)$ corrections made for a putative metallicity-dependency to the PL relations - i.e., $\gamma_{\mathrm{VI}}=-0.2 \mathrm{mag} \mathrm{dex}^{-1}$ and $\gamma_{\mathrm{VI}}=+0.0 \mathrm{mag} \mathrm{dex}^{-1}$, respectively - were undertaken. The number of Cepheids employed in the fitting and the accompanying total random uncertainties for each galaxy are also listed.

Table 1. Distances to Nearby Type Ia Supernovae Luminosity Calibrators and their Host Galaxies

\begin{tabular}{llcccc}
\hline Galaxy & Supernova & $\mathrm{n}_{\text {Ceph }}{ }^{a}$ & $\mu_{\circ}{ }^{b}$ & $\mu_{\mathrm{Z}}{ }^{c}$ & $\sigma_{\mu}(\mathrm{r})^{d}$ \\
\hline NGC 4527 & $1991 \mathrm{~T}$ & 16 & 30.482 & 30.562 & \pm 0.085 \\
NGC 4639 & $1990 \mathrm{~N}$ & 17 & 31.524 & 31.624 & \pm 0.084 \\
NGC 4536 & $1981 \mathrm{~B}$ & 27 & 30.693 & 30.763 & \pm 0.069 \\
NGC 3627 & 1989B & 17 & 29.794 & 29.944 & \pm 0.169 \\
NGC 3368 & $1998 \mathrm{bu}$ & 7 & 29.956 & 30.096 & \pm 0.098 \\
NGC 5253 & 1972E & 7 & 27.485 & 27.415 & \pm 0.119 \\
IC 4182 & 1937C & 28 & 28.207 & 28.187 & \pm 0.076 \\
\hline \hline
\end{tabular}

${ }^{a}$ Number of Cepheids employed in PL fitting. The Cepheids are identical to those used by Gibson et al. (2000) and Gibson \& Stetson (2001).

${ }^{b}$ True distance moduli to Type Ia SN host galaxies, assuming Stetson (1998) WFPC photometric calibration, Udalski et al. (1999) LMC PL slopes and apparent zero points, and referenced to an LMC distance of $49 \mathrm{kpc}$.

${ }^{c}$ Metallicity-corrected distance moduli, assuming $\gamma_{\mathrm{VI}}=-0.2 \mathrm{mag} \mathrm{dex}^{-1}$ and Cepheid field metallicities as listed in Gibson et al. (2000; Table 1) and Gibson \& Stetson (2001).

${ }^{d}$ Random uncertainties are derived following Gibson et al. (2000), and correspond to item $R_{\mathrm{PL}}$ of Table 7 therein.

Using the SN photometry and reddenings tabulated by Gibson et al. (2000; Table 5), supplemented now with the apparent peak magnitudes (Lira et al. 1998; Table 7) and Galactic+intrinsic reddenings (Phillips et al. 1999; Table 2) for SN 1991T, the B-, V-, and I-band peak luminosities for the seven calibrating $\mathrm{SNe}$ were then calculated. While omitted for brevity's sake, they are listed in columns 3-5 of Table 3 in Gibson \& Stetson (2001), assuming the metallicity-corrected true moduli $\mu_{\mathrm{Z}}$ listed in Table 1 . It is important to note that the corrected peak luminosity for SN $1991 \mathrm{~T}$ (e.g., $\mathrm{M}_{\mathrm{B}, \mathrm{mar}}^{\max }=-19.40 \pm 0.24$ ) is indistinguishable from that of the mean of the full sample of nine calibrators $\left(<\mathrm{M}_{\mathrm{B}, \mathrm{morr}}^{\max }>=-19.32 \pm 0.08\right)$. While $\mathrm{SN} 1991 \mathrm{~T}$ is only one datum, there exists no evidence to suggest that spectroscopically peculiar Type Ia SNe need be dismissed a priori from future extragalactic distance scale work.

The calibrated (corrected) peak luminosities can then be used in conjunction with either the quadratic or linear fits presented in Figure 1 (in conjunction with equations 1 and 4) to provide colour-dependent Hubble Constants for each of the seven calibrators. The weighted mean of $\mathrm{H}_{0}(B), \mathrm{H}_{0}(\mathrm{~V})$, and $\mathrm{H}_{0}(\mathrm{I})$ yields 
$\mathrm{H}_{0}=73 \mathrm{~km} \mathrm{~s}^{-1} \mathrm{Mpc}^{-1}$, with a total random uncertainty of $\pm 2 \mathrm{~km} \mathrm{~s}^{-1} \mathrm{Mpc}^{-1}$ both the quadratic and linear fits of Figure 1 lead to the identical result. f

After Freedman et al. (2001), seven sources of error were incorporated into the systematic error budget (and are listed in Table 2). Uncertainties in the LMC zero point, crowding, and large scale bulk flows each enter in at the $\pm 0.10 \mathrm{mag}$ level; the metallicity dependency of the Cepheid PL relation at the $\pm 0.08 \mathrm{mag}$ level; the WFPC2 zero point uncertainty at the \pm 0.07 mag level; reddening and bias in the Cepheid PL fitting at the $\pm 0.02 \mathrm{mag}$ level each. In quadrature, the overall systematic error budget amounts to $0.21 \mathrm{mag}$, corresponding to $10 \%$ in $\mathrm{H}_{0}$. Significantly improving the precision to which we can derive $\mathrm{H}_{0}$ via Cepheid calibration of secondary distance indicators will require a factor of two reduction in uncertainty in each of these five remaining dominant sources of systematic uncertainty. Until then, we are limited to $10 \%$ precision.

Table 2. Systematic Error Budget

\begin{tabular}{lc}
\hline Source of Uncertainty & Error (mag) \\
\hline LMC true modulus & \pm 0.10 \\
Stellar profile crowding on WFPC2 & \pm 0.10 \\
Large-scale bulk flows & \pm 0.10 \\
Cepheid PL-[O/H] sensitivity & \pm 0.08 \\
WFPC2 calibration & \pm 0.07 \\
Cepheid reddening & \pm 0.02 \\
Bias in Cepheid PL relation & \pm 0.02 \\
Total Systematic Uncertainty & \pm 0.21 \\
\hline \hline
\end{tabular}

In combination, the above random (r) and systematic (s) error budget yields a final result for the Hubble Constant of

$$
\mathrm{H}_{0}=73 \pm 2(\mathrm{r}) \pm 7(\mathrm{~s}) \mathrm{km} \mathrm{s}^{-1} \mathrm{Mpc}^{-1} .
$$

Ignoring the metallicity dependency in the Cepheid PL relation (i.e., using $\gamma_{\mathrm{VI}}=+0.0$ mag dex ${ }^{-1}$, as opposed to the $\gamma_{\mathrm{VI}}=-0.2 \pm 0.2 \mathrm{mag} \mathrm{dex}^{-1}$ employed here) increases $\mathrm{H}_{0}$ by $3 \mathrm{~km} \mathrm{~s}^{-1} \mathrm{Mpc}^{-1}$.

As emphasised earlier, a variety of fits to C-T and CfA SNe samples were undertaken. The canonical linear and quadratic fits in $\Delta m_{15}$ incorporated (i) the Schlegel et al. (1998) and Phillips et al. (1999) prescriptions for foreground and host galaxy reddening, respectively; (ii) extinction-dependent corrections to the decline rate, also after Phillips et al. (1999); (iii) recessional velocity uncertainties of $\pm 600 \mathrm{~km} \mathrm{~s}^{-1}$; (iv) ratios of total-to-selective absorption of $R_{B}: R_{V}: R_{I}=4.300: 3.300: 1.964$. In addition, the combined dataset was culled from 51 to 36 (32 for the I-band), to avoid highly-reddened and low-velocity

\footnotetext{
${ }^{4}$ The formal difference is at the $<0.7 \%$ level - i.e., $<0.2 \sigma$ !
} 
(where the peculiar velocity uncertainty becomes a substantial fraction of the recessional velocity itself) interlopers biasing the analysis.

The colour-dependent coefficients provided by both the bivariate linear least squares and quadratic bootstrapping fits in light curve decline rate are robust against the adopted extinction law, extinction-dependent corrections to the light curve shape, and host galaxy peculiar velocity uncertainty. The only two permutations which impacted equation (5) at more than the $1 \%$ level were: (i) restricting the analysis to the $26 \mathrm{C}-\mathrm{T}$ SNe alone (as opposed to the combined default sample of 36 ) reduces $\mathrm{H}_{0}$ by $\sim 2 \%$ (i.e., $\sim 1 \sigma$ ); (ii) completely neglecting the host galaxy reddening correction (in both the C-T+CfA and nearby calibrators) increases $\mathrm{H}_{0}$ by $\sim 4 \%$ (i.e., $\sim 2 \sigma$ ). The poor quality of the linear and

quadratic fits for case (ii) - e.g., the B-band dispersion increases from $\sim 0.14 \mathrm{mag}$ to $\sim 0.21 \mathrm{mag}$ - makes the significance of this latter effect questionable at best.

\section{Summary}

A re-analysis of the combined Calán-Tololo and Center for Astrophysics Type Ia $\mathrm{SNe}$ datasets is presented, with new linear and quadratic fits to the empirical peak magnitude-decline rate relationship determined. The fit coefficients are extremely robust to uncertainties in photometry, reddening, and peculiar velocity. With a calibration to the corrected Hubble Diagrams now provided by seven high-quality nearby calibrators with accurate host galaxy Cepheid distance determinations, our favoured value for the Hubble Constant becomes:

$$
\mathrm{H}_{0}=73 \pm 2(\mathrm{r}) \pm 7(\mathrm{~s}) \mathrm{km} \mathrm{s}^{-1} \mathrm{Mpc}^{-1}
$$

The systematic uncertainties clearly limit the precision to which we can determine $\mathrm{H}_{0}$; until significant progress is made in improving our understanding of, for example, the distance to the LMC and the sensitivity of the Cepheid PL relation to metallicity, the uncertainty associated with Cepheid-based Hubble Constant cannot be reduced below $10 \%$.

Acknowledgments. We wish to thank Peter Stetson for his crucial contributions throughout the course of this work.

\section{References}

Burstein, D. \& Heiles, C. 1982, AJ, 87, 1165

Dolphin, A.E. 2000, PASP, 112, 1397

Freedman, W.L., Madore, B.F., Gibson, B.K., et al. 2001, submitted

Gibson, B.K., Stetson, P.B., Freedman, W.L., et al. 2000, ApJ, 529, 723

Gibson, B.K. \& Stetson, P.B. 2001, submitted

Hamuy, M., Phillips, M.M., Schommer, R.A., et al. 1996, AJ, 112, 2391

Hill, R.J., et al. 1998, ApJ, 496, 648

Höflich, P. \& Khokhlov, A. 1996, ApJ, 457, 500

Jha, S., Garnavich, P.M., Kirshner, R.P., et al. 1999, ApJS, 125, 73

Lira, P., Hamuy, M., Wells, L.A., et al. 1998, AJ, 115, 234 
Madore, B.F. \& Freedman, W.L. 1991, PASP, 103, 933

Phillips, M.M., Lira, P., Suntzeff, N.B., et al. 1999, AJ, 118, 1766

Phillips, M.M. 2000, http://www.lco.cl/users/mmp/reddening/SNeIa_mags.htm]

Riess, A.G., Press, W.H. \& Kirshner, R.P. 1996, ApJ, 473, 88

Riess, A.G., Filippenko, A.V., Challis, P., et al. 1998, AJ, 116, 1009

Saha, A., Sandage, A., Tammann, G.A., et al. 1999, ApJ, 522, 802

Stetson, P.B. 1994, AJ, 106, 205

Stetson, P.B. 1996, PASP, 108, 851

Stetson, P.B. 1998, PASP, 110, 1448

Suntzeff, N.B., Phillips, M.M., Covarrubias, R., et al. 1999, AJ, 117, 1175

Tanvir, N.R., Ferguson, H.C. \& Shanks, T. 1999, MNRAS, 310, 175

Turner, A., Ferrarese, L., Saha, A., et al. 1998, ApJ, 505, 207

Udalski, A., Szymański, M., Kubiak, M., et al. 1999, AcA, 49, 201

Whitmore, B., Heyer, I. \& Casertano, S. 1999, PASP, 111, 1559 